\title{
ON A CLASS OF GENERALIZED FERMAT EQUATIONS
}

\author{
ANDRZEJ DĄBROWSKI
}

(Received 22 November 2009)

\begin{abstract}
We generalize the main result of the paper by Bennett and Mulholland ['On the diophantine equation $x^{n}+y^{n}=2^{\alpha} p z^{2}$, C. R. Math. Acad. Sci. Soc. R. Can. 28 (2006), 6-11] concerning the solubility of the diophantine equation $x^{n}+y^{n}=2^{\alpha} p z^{2}$. We also demonstrate, by way of examples, that questions about solubility of a class of diophantine equations of type $(3,3, p)$ or $(4,2, p)$ can be reduced, in certain cases, to studying several equations of the type $(p, p, 2)$.
\end{abstract}

2000 Mathematics subject classification: primary 11D41; secondary 11F33, 11F80, $11 \mathrm{G} 05$.

Keywords and phrases: generalized Fermat equation, elliptic curve, modular form, Galois representation.

\section{Introduction}

By the work of Hellegouarch, Frey, Serre, Ribet, Wiles, Taylor and many others [5, 13$15]$, we can reduce the study of a class of ternary diophantine equations (generalized Fermat equations) $A x^{p}+B y^{q}=C z^{r}$ to modern techniques coming from Galois representations and modular forms. In all known cases, the proofs follow a variant of the method of Frey (or Frey-Hellegouarch) curves and Ribet's levellowering theorem. We should stress that Frey curves have been constructed for only a few families of diophantine equations. In particular, a number of partial (sometimes complete) results are available when $(p, q, r)$ is one of the following types: $(p, p, p),(p, p, 2),(p, p, 3),(3,3, p),(4,4, p),(5,5, p),(2,4, p)$.

In this paper we prove the following result, generalizing [1, Theorem 1.1].

THEOREM 1.1. Let $M$ be an odd squarefree positive integer, $\operatorname{gcd}(M, 21)=1$. Then the equation

$$
x^{n}+y^{n}=2^{\alpha} M z^{2}
$$

has no solutions in coprime nonzero integers $x$ and $y$, positive integers $z$ and $\alpha$, and primes $n$ satisfying $n>M^{132 M^{2}}$.

Similarly, one can generalize the results from the paper by Bennett et al. [3] (see the remark in Section 3).

(C) 2010 Australian Mathematical Publishing Association Inc. 0004-9727/2010 \$16.00 
In Sections 4, 5 and 6 we give new proofs of results concerning solubility of specific diophantine equations of types $(3,3, p)$ and $(4,2, p)$. In these cases we reduce the problem to studying several diophantine equations of type $(p, p, 2)$.

\section{Preliminaries}

LEMMA 2.1. Suppose that $p \geq 7$ is prime.

(i) If $\left(C, \alpha_{0}\right) \in\{(1,2),(3,2)\}$, then the equation $x^{p}+2^{\alpha} y^{p}=C z^{2}$ has no solutions in nonzero pairwise coprime integers $(x, y, z)$ with $x y \neq \pm 1$ and integers $\alpha \geq \alpha_{0}$.

(ii) If $C \in\{1, \overline{2}\}$, then the equation $x^{p}+y^{p}=C z^{2}$ has no solutions in nonzero coprime integers $(x, y, z)$ with $x y \neq \pm 1$.

PROOF. Special cases of Theorems 1.2 and 1.1 in [2]. See also [7, Main theorem].

LEMMA 2.2. For non-zero integers $x$, $y$ satisfying $\operatorname{gcd}(x, y)=1$, we have:

(i) $\operatorname{gcd}\left(x+y, x^{2}+y^{2}\right)=1$ or 2 ;

(ii) $\operatorname{gcd}\left(x+y, x^{2}-x y+y^{2}\right)=1$ or 3 .

PROOF. (i) Assume $r^{e} \mid x+y$ and $r^{e} \mid x^{2}+y^{2}$, where $r$ is a prime and $e$ is a positive integer. Then $r^{e} \mid 2 x^{2}$. But $\operatorname{gcd}(x, y)=1$, hence $r \nmid x$. Therefore $r^{e} \mid 2$ and the assertion follows. The proof of (ii) follows along the same lines.

\section{Generalization of Bennett and Mulholland's result}

The proof of Theorem 1.1 follows along the same lines as the proof of [1, Theorem 1.1], therefore we only indicate the main steps. The genuine new ingredient is Lemma 3.1 below. The point is that a classification of elliptic curves over $\mathbb{Q}$ with rational 2-torsion point and conductor $32 M^{2}$ or $256 M^{2}$ is not necessary-here we use a much weaker result.

Let

$$
E=E(a, b, c): Y^{2}=X^{3}+2^{\beta+1} c M X^{2}+2^{\beta} M b^{n} X
$$

denote the elliptic curve attached to nontrivial solution of (1.1). Let $\rho_{n}^{E}$ denote the corresponding $\bmod n$ Galois representation. Using [2, Lemmas 3.2 and 3.3] we obtain that this representation arises from a cuspidal newform of weight 2 , trivial Nebentypus, and level $32 M^{2}$ or $256 M^{2}$. Let $f$ be a cuspidal newform of weight 2 , level $N$, and trivial Nebentypus, where $N=32 M^{2}$ or $256 M^{2}$.

If $f$ has at least one Fourier coefficient that is not a rational integer, then we obtain (analogously to [1]) $n \leq M^{12 M^{2}}$ if $N=32 M^{2}$, and $n \leq M^{132 M^{2}}$ if $N=256 M^{2}$.

If $f$ has only rational integer Fourier coefficients, then we argue as in [1], replacing Propositions 3.1 and 3.2 by the following result.

LEMMA 3.1. Let $E$ be an elliptic curve defined over $\mathbb{Q}$ with rational 2-torsion and conductor $32 M^{2}$ or $256 M^{2}$, where $M$ is an odd squarefree integer. If $\operatorname{gcd}(M, 21)=1$, then $E$ has $j$-invariant whose denominator is divisible by some prime $p_{0} \mid M$ or $C M$ by an order in $\mathbb{Q}(\sqrt{-1})$ or $\mathbb{Q}(\sqrt{-2})$. 
PRoOF. Write $M=p_{1} \cdots p_{k}$. Generalizing [8, Lemme 1], we deduce that $E$ has global minimal model of the form

$$
y^{2}=x\left(x^{2}+a x+b\right),
$$

with integers $a, b \in \mathbb{Z}$ without common prime factors different from $2, p_{1}, \ldots, p_{k}$. One can easily check that

$$
c_{4}=2^{4}\left(a^{2}-3 b\right), \quad c_{6}=2^{5} a\left(9 b-2 a^{2}\right), \quad \Delta_{E}=2^{4} b^{2}\left(a^{2}-4 b\right) .
$$

If $a=0$, then $E$ has CM by $\mathbb{Q}(\sqrt{-1})$.

Assume that $a \neq 0$, and write

$$
\Delta_{E}= \pm 2^{m} p_{1}^{\alpha_{1}} \cdots p_{k}^{\alpha_{k}}, \quad a= \pm 2^{m_{1}} p_{1}^{\beta_{1}} \cdots p_{k}^{\beta_{k}} a_{0}, \quad b= \pm 2^{m_{2}} p_{1}^{\gamma_{1}} \cdots p_{k}^{\gamma_{k}} .
$$

Using [12, Tableau IV] we obtain

$$
\begin{aligned}
& \left(v_{2}\left(\Delta_{E}\right), v_{2}\left(c_{4}\right), v_{2}\left(c_{6}\right)\right) \\
& \quad \in\{(6,4, \geq 6),(9,4,6),(12,6, \geq 9),(12,7,9)\} \quad \text { if } N_{E}=32 M^{2},
\end{aligned}
$$

and

$$
\left(v_{2}\left(\Delta_{E}\right), v_{2}\left(c_{4}\right), v_{2}\left(c_{6}\right)\right) \in\{(9,5, \geq 8),(15,7, \geq 11)\} \quad \text { if } N_{E}=256 M^{2} .
$$

If $2 \beta_{i_{0}}<\gamma_{i_{0}}$ for some $i_{0} \in\{1, \ldots, k\}$, then the denominator of $j_{E}$ is divisible by $p_{i_{0}}$.

If $2 \beta_{i} \geq \gamma_{i}$ for all $i \in\{1, \ldots, k\}$, then careful analysis of possible cases for $\left(v_{2}\left(\Delta_{E}\right), v_{2}\left(c_{4}\right), v_{2}\left(c_{6}\right)\right)$ leads to elliptic curves with CM by $\mathbb{Q}(\sqrt{-1})$ or $\mathbb{Q}(\sqrt{-2})$, or to elliptic curves with $j$-invariants whose denominators are divisible by some prime $p_{0} \mid M$. Let us give some details (possible values of $\left(m_{1}, m_{2}\right)$ will follow from the formulas for $c_{4}, c_{6}$ and $\Delta_{E}$, given above).

$\left(v_{2}\left(\Delta_{E}\right), v_{2}\left(c_{4}\right), v_{2}\left(c_{6}\right)\right)=(6,4, \geq 6)$. In this case $\left(m_{1}, m_{2}\right)=(0,1)$ or $(>1,0)$. If $\left(m_{1}, m_{2}\right)=(0,1)$, then denominator of $j_{E}$ is divisible by some $p_{0} \mid M$, or $p_{1}^{2 \beta_{1}-\gamma_{1}} \cdots p_{k}^{2 \beta_{k}-\gamma_{k}} a_{0}^{2} \pm 8= \pm 1$. In the second case $a_{0}= \pm 3$ and $\gamma_{i}=2 \beta_{i}$ for all $i \in\{1, \ldots, k\}$ (here we use the assumption $\operatorname{gcd}\left(p_{i}, 21\right)=1$ ), and we obtain a family of elliptic curves $y^{2}=x^{3} \pm 3 M x^{2}+2 M^{2} x$ with CM by $\mathbb{Q}(\sqrt{-1})$. The case $\left(m_{1}, m_{2}\right)=(>1,0)$ leads to elliptic curves with $j$-invariants whose denominators are divisible by some $p_{0} \mid M$.

$\left(v_{2}\left(\Delta_{E}\right), v_{2}\left(c_{4}\right), v_{2}\left(c_{6}\right)\right)=(9,4,6)$. In this case $\left(m_{1}, m_{2}\right)=(1,0)$, and we obtain elliptic curves with $j$-invariants whose denominators are divisible by some $p_{0} \mid M$, or a family $y^{2}=x^{3} \pm 3 M x^{2}+2 M^{2} x$ with CM by $\mathbb{Q}(\sqrt{-1})$.

$\left(v_{2}\left(\Delta_{E}\right), v_{2}\left(c_{4}\right), v_{2}\left(c_{6}\right)\right)=(9,5, \geq 8)$. In this case $\left(m_{1}, m_{2}\right)=(\geq 2,1)$, and we obtain elliptic curves with $j$-invariants whose denominators are divisible by some $p_{0} \mid M$, or a family $y^{2}=x^{3} \pm 4 M x^{2}+2 M^{2} x$ with CM by $\mathbb{Q}(\sqrt{-2})$.

$\left(v_{2}\left(\Delta_{E}\right), v_{2}\left(c_{4}\right), v_{2}\left(c_{6}\right)\right)=(12,6, \geq 9)$. In this case $\left(m_{1}, m_{2}\right)=(1,3)$, and we obtain elliptic curves with $j$-invariants whose denominators are divisible by some $p_{0} \mid M$, or a family $y^{2}=x^{3} \pm 3 M x^{2}+2 M^{2} x$ with CM by $\mathbb{Q}(\sqrt{-1})$. 
$\left(v_{2}\left(\Delta_{E}\right), v_{2}\left(c_{4}\right), v_{2}\left(c_{6}\right)\right)=(12,7,9)$. This case produces no elliptic curve. Indeed, $m_{1} m_{2}=0$ implies $v_{2}\left(c_{4}\right)=4$ or $v_{2}\left(\Delta_{E}\right)=4$, a contradiction. Let $m_{1} m_{2} \geq 1$. Then $m_{1}=1$ implies $m_{2}=2$, and hence $v_{2}\left(c_{6}\right)=8$; similarly, $m_{1} \geq 2$ implies $m_{2}=3$, and hence $v_{2}\left(c_{6}\right) \geq 10$.

$\left(v_{2}\left(\Delta_{E}\right), v_{2}\left(c_{4}\right), v_{2}\left(c_{6}\right)\right)=(15,7, \geq 11)$. In this case $\left(m_{1}, m_{2}\right)=(\geq 3,3)$, and we obtain elliptic curves with $j$-invariants whose denominators are divisible by some $p_{0} \mid M$, or a family $y^{2}=x^{3} \pm 8 M x^{2}+8 M^{2} x$ with CM by $\mathbb{Q}(\sqrt{-2})$.

REMARK 3.2. One can generalize [3, Theorems 1.1, 1.3 and 1.4]: here we replace Proposition 6.1 by a variant of Lemma 3.1. It is clear that variants of Lemma 3.1 will apply to some other types of generalized Fermat equations.

\section{New proof of Billerey's result}

Let $p$ be an odd prime. Consider the equation

$$
(x+y)\left(x^{2}+y^{2}\right)=z^{p}, \quad \operatorname{gcd}(x, y)=1 .
$$

By Lemma 2.2 we have two cases to consider.

(i) Assume that $\operatorname{gcd}\left(x+y, x^{2}+y^{2}\right)=2$. In this case $x+y=2^{p-1} z_{1}^{p}$ and $x^{2}+$ $y^{2}=2 z_{2}^{p}$, with $\operatorname{gcd}\left(z_{1}, z_{2}\right)=1$. Substituting $y=-x+2^{p-1} z_{1}^{p}$ in the second equation we obtain

$$
2 x^{2}-2^{p} z_{1}^{p} x+2^{2 p-2} z_{1}^{2 p}-2 z_{2}^{p}=0 .
$$

We have $\Delta_{x}=16\left(z_{2}^{p}-2^{2 p-4} z_{1}^{2 p}\right)$. Using Lemma 2.1(i), we obtain that the equation $X^{p}+2^{m} Y^{p}=Z^{2}(m \geq 2)$ has no solution in nonzero pairwise coprime integers $(X, Y, Z)$ with $X Y \neq 1$. As a corollary we obtain the following result [4, Theorem 3.1].

Proposition 4.1. Equation (4.1) has no nontrivial solution in integers $x, y, z$ with z even.

(ii) Assume that $\operatorname{gcd}\left(x+y, x^{2}+y^{2}\right)=1$. In this case $x+y=z_{1}^{p}$ and $x^{2}+$ $y^{2}=z_{2}^{p}$, with $\operatorname{gcd}\left(z_{1}, z_{2}\right)=1$. Substituting $y=-x+z_{1}^{p}$ in the second equation we obtain $2 x^{2}-2 z_{1}^{p} x+z_{1}^{2 p}-z_{2}^{p}=0$. We have $\Delta_{x}=4\left(2 z_{2}^{p}-z_{1}^{2 p}\right)$. It is expected that the equation $2 X^{p}+Y^{p}=Z^{2}, \operatorname{gcd}(X, Y)=1$, has no solutions in nonzero coprime integers $(X, Y, Z)$ with $X Y \neq \pm 1$, and hence (4.1) has no solutions. Such an expectation follows from [9, Conjecture 2], at least for $p$ sufficiently large.

\section{Application to the equation $x^{3}+y^{3}=z^{p}$}

Let $p$ be an odd prime. Consider the equation

$$
x^{3}+y^{3}=z^{p}, \quad \operatorname{gcd}(x, y)=1 .
$$

Assume that $p \geq 17$ and $(a, b, c)$ is a nontrivial solution to Equation (5.1), satisfying $a c$ even. Kraus [11, Theorem 6.1] has proved the following result. 
PROPOSITION 5.1.

(i) $c$ is odd;

(ii) $v_{2}(a)=1$;

(iii) $v_{3}(c) \geq 1$.

We give another proof of this result. By Lemma 2.2 we have two cases to consider.

(i) Assume that $\operatorname{gcd}\left(x+y, x^{2}-x y+y^{2}\right)=1$. In this case $x+y=z_{1}^{p}$ and $x^{2}-x y+y^{2}=z_{2}^{p}$, with $\operatorname{gcd}\left(z_{1}, z_{2}\right)=1$. Substituting $y=-x+z_{1}^{p}$ in the second equation, we obtain $3 x^{2}-3 z_{1}^{p} x+z_{1}^{2 p}-z_{2}^{p}=0$. We have $\Delta_{x}=3\left(4 z_{2}^{p}-z_{1}^{2 p}\right)$. Using Lemma 2.1(i), we obtain that the equation $4 X^{p}+Y^{p}=3 Z^{2}, \operatorname{gcd}(X, Y)=1$, has no nontrivial solution in integers satisfying $X Y \neq \pm 1$. In particular, Equation (5.1) has no solution if $z$ is even. This proves case (i).

(ii) Assume that $\operatorname{gcd}\left(x+y, x^{2}-x y+y^{2}\right)=3$. Then, in particular, $v_{3}(z) \geq 1$. In this case we have $x+y=3^{p-1} z_{1}^{p}$ and $x^{2}-x y+y^{2}=3 z_{2}^{p}$, with $\operatorname{gcd}\left(z_{1}, z_{2}\right)=1$. Substituting $y=-x+3^{p-1} z_{1}^{p}$, we arrive at the diophantine equation $4 z_{2}^{p}-$ $3^{2 p-3} z_{1}^{p}=t^{2}$. Here we are in case (iii) from [2]: $A=3^{p-3}, B=4, C=1$. Let $E=E_{3}(a, b, c)$ be the corresponding elliptic curve. Using [2, Lemma 3.3], we obtain that the corresponding Galois representation $\rho_{E, p}$ (with $p \geq 7$ ) arises from a cuspidal newform of weight 2 and level 12 (level 24) if $z_{2} \equiv 3 \bmod 4\left(z_{2} \equiv 1 \bmod 4\right)$. There are no nonzero cuspforms of weight 2 and level 12. In the case of level 24 we have $z_{2} \equiv 1 \bmod 4$, hence $x \equiv 2 \bmod 4$, proving case (ii).

REMARKS 5.2.

(i) Note that $3^{p-3} \pm 4$ are not squares of integers. Therefore [9, Conjecture 1] implies that the equation $4 X^{p}+3^{p-3} Y^{p}=Z^{2}$ has no nontrivial solutions. Consequently (5.1) has no nontrivial solutions.

(ii) Kraus [11] showed that (5.1) has no nontrivial solutions for exponents $p$ with $17 \leq p \leq 10^{4}$; the same can be proved for $5 \leq p \leq 13$.

\section{Application to the equation $x^{4}-y^{2}=n z^{p}$}

Let $p \geq 5$ be prime and $n$ a positive integer greater than 1. Dąbrowski [6] proves that, under certain conditions on $n$, the equation $x^{4}-y^{4}=n z^{p}$ has no nontrivial solution in $\mathbb{Z}$ if $p \geq C(n)$, where $C(n)$ is effectively a constant. Let us state a particular case [6, Corollary 1].

Proposition 6.1. Let $q$ be an odd prime, not of the type $2^{m} \pm 1$. Let $p$ be a prime satisfying $p>(\sqrt{8 q+8}+1)^{2 q-2}$. Then the equation $x^{4}-y^{4}=q z^{p}$ has no nontrivial solution in the integers.

We will deduce the following version of this result from [9]. We should stress that both proofs use ideas from [10].

Proposition 6.2. Let $q>3$ be a prime; assume that $q \equiv 3 \bmod 8$ and $q \neq 2 t^{2}+1$, or $q \equiv 5 \bmod 8$ and $q \neq t^{2}+4$. In addition, let $p$ be a prime satisfying $p>(8 \sqrt{q+1}+1)^{16(q-1)}$. Then the equation $x^{4}-y^{2}=q z^{p}$ has no nontrivial solution in the integers. 
PROOF. The case where $x y$ is even leads to consideration of the diophantine equation $q X^{p}+Y^{p}=2 Z^{2}$. Theorem 1.2 in [9] implies that it has no nontrivial solution if $q \equiv 3 \bmod 8$ and $q \neq 2 t^{2}+1$ or $q \equiv 5 \bmod 8$, and $p>(8 \sqrt{q+1}+1)^{16(q-1)}$.

The case where $x y$ is odd leads to consideration of two diophantine equations:

(i) $X^{p}+4 q Y^{p}=Z^{2}$;

(ii) $4 X^{p}+q Y^{p}=Z^{2}$.

Theorem 1.1 in [9] implies that these equations have no nontrivial solution if $q \equiv$ $3 \bmod 8$ or $q \equiv 5 \bmod 8$ and $q \neq t^{2}+4$, and $p>(8 \sqrt{q+1}+1)^{16(q-1)}$.

REMARK 6.3. Some questions concerning solubility of a general diophantine equation $x^{4}-y^{2}=n z^{p}$ may be reduced to [9, Conjectures 1 and 2].

\section{References}

[1] M. A. Bennett and J. Mulholland, 'On the diophantine equation $x^{n}+y^{n}=2^{\alpha} p z^{2}$, C. R. Math. Acad. Sci. Soc. R. Can. 28 (2006), 6-11.

[2] M. A. Bennett and C. M. Skinner, 'Ternary diophantine equations via Galois representations and modular forms', Canad. J. Math. 56 (2004), 23-54.

[3] M. A. Bennett, V. Vatsal and S. Yazdani, 'Ternary diophantine equations of signature $(p, p, 3)$ ', Compositio Math. 140 (2004), 1399-1416.

[4] N. Billerey, 'Formes homogènes de degré 3 et puissances $p$-ièmes', J. Number Theory 128 (2008), 1272-1294.

[5] C. Breuil, B. Conrad, F. Diamond and R. Taylor, 'On the modularity of elliptic curves over $\mathbb{Q}$ : wild 3-adic exercises', J. Amer. Math. Soc. 14 (2001), 843-939.

[6] A. Dąbrowski, 'On the integers represented by $x^{4}-y^{4}$, Bull. Aust. Math. Soc. 76 (2007), 133-136.

[7] H. Darmon and L. Merel, 'Winding quotients and some variants of Fermat's last theorem', J. reine angew. Math. 490 (1997), 81-100.

[8] W. Ivorra, 'Courbes elliptiques sur $\mathbb{Q}$, ayant un point d'ordre 2 rationnel sur $\mathbb{Q}$, de conducteur $2^{N}$ p', Dissert. Math. 429 (2004), 55pp.

[9] W. Ivorra and A. Kraus, 'Quelques résultats sur les équations $a x^{p}+b y^{p}=c z^{2}$, Canad. J. Math. $\mathbf{5 8}(2006), 115-153$.

[10] A. Kraus, 'Majorations effectives pour l'équation de Fermat généralisée', Canad. J. Math. 49 (1997), 1139-1161.

[11] A. Kraus, 'Sur l'équation $a^{3}+b^{3}=c^{p}$ ', Experiment Math. 7 (1998), 1-13.

[12] I. Papadopoulos, 'Sur la classification de Néron des courbes elliptiques en caractéristique résiduelle 2 et 3', J. Number Theory 44 (1993), 119-152.

[13] K. Ribet, 'On modular representations of $\mathrm{Gal}(\overline{\mathbf{Q}} / \mathbf{Q})$ arising from modular forms', Invent. Math. 100 (1990), 431-476.

[14] J.-P. Serre, 'Sur les représentations modulaires de degré 2 de Gal $(\overline{\mathbf{Q}} / \mathbf{Q})$ ', Duke Math. J. 54 (1987), 179-230.

[15] A. Wiles, 'Modular elliptic curves and Fermat's last theorem', Ann. of Math. (2) 141 (1995), 443-551.

\section{ANDRZEJ DĄBROWSKI, Institute of Mathematics, University of Szczecin, ul. Wielkopolska 15, 70-451 Szczecin, Poland e-mail: dabrowsk@wmf.univ.szczecin.pl}

